EPJ Web of Conferences 41, 02031 (2013)

DOI: $10.1051 /$ epjconf/20134102031

(C) Owned by the authors, published by EDP Sciences, 2013

\title{
Initial Processes of Proton Transfer in Salicylideneaniline Studied by Time-Resolved Photoelectron Spectroscopy
}

\author{
Taro Sekikawa, ${ }^{1,2}$ Oliver Schalk, ${ }^{2,3}$ Guorong Wu, ${ }^{2}$ Andrey E. Boguslavskiy, ${ }^{2}$ and Albert Stolow ${ }^{2}$ \\ ${ }^{1}$ Department of Applied Physics, Hokkaido University, Sapporo, 060-8628 Japan \\ ${ }^{2}$ National Research Council Canada, Ottawa, Ontario K1A 0R6 Canada \\ ${ }^{3}$ Lehrstuhl für BioMolekulare Optik, Ludwig-Maximilians Universität, 80538 München, Germany
}

\begin{abstract}
Excited state intramolecular proton transfer (ESIPT) in salicylideneaniline (SA) and selected derivatives substituted in para-position of the anilino group has been investigated by femtosecond time-resolved photoelectron spectroscopy (TRPES) and time-dependent density functional theory (TDDFT). The planarity of the molecule was found to be a key parameter to describe ESIPT.
\end{abstract}

\section{Introduction}

Proton transfer is a fundamental and important chemical reaction in biological systems. To better understand this process in complex biological surroundings, one fruitful approach is to start the investigation in a simpler molecular system. Here, we study the excited state proton transfer (ESIPT) reaction in salicylideneaniline (SA). SA (see Fig. 1) has been one of the best characterized molecules so far. The basic understanding of the mechanism is as follows: In the ground state, the enol form is more stable than the keto form. On the other hand, the proton-transferred cis-keto form is more stable in the excited states. Thus, upon photoexcitation, the proton of the hydrogen bond is pushed toward the nitrogen forming the cis-keto form. Subsequently, cis-trans isomerization takes place and brings the molecule back to the ground state in its photochromic product, the trans-keto form from where it eventually returns to the enol form by ground state processes.

However, advances of experimental and theoretical investigations revealed that the relaxation

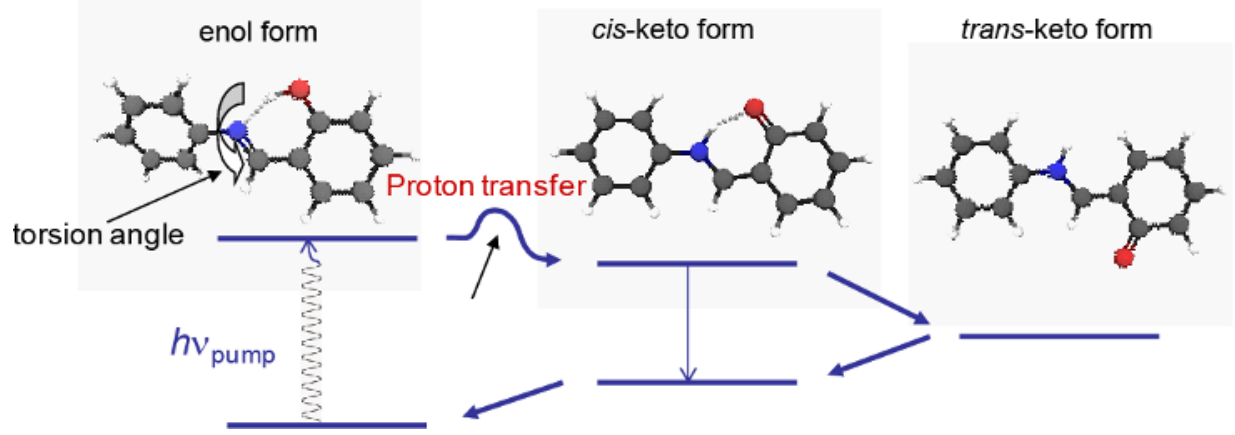

Fig. 1 : Scheme of the relaxation processes of photo-excited salicylideneaniline

This is an Open Access article distributed under the terms of the Creative Commons Attribution License 2.0, which permits unrestricted use, distribution, and reproduction in any medium, provided the original work is properly cited. 
process is more complicated, e.g., the aromatic groups are able to rotate and one potentially minor relaxation path was found to follow rotation of these groups in the enol-form [1]. To gain insight into the intrinsic nature of the proton transfer dynamics, we thought it is useful to investigate solvent-free molecules as they are provided by supersonic expansion. As spectroscopic method, we chose timeresolved photoelectron spectroscopy (TRPES).

\section{Experiment}

Our experimental setup for TRPES was as follows: The output of Ti:sapphire laser was separated into two beams. One was used to pump an optical parametric amplifier to generate IR-pulses around $1400 \mathrm{~nm}$. The frequency of the output beam was doubled twice by nonlinear crystals to generate $\sim 350$-nm pulses, which were used as pump beam. The pump wavelengths were 370, 350 and $330 \mathrm{~nm}$. As probe pulses we used the second harmonic of the second beam at $400 \mathrm{~nm}$. Upon photoexcitation, SA molecules were ionized via two-photon absorption. The temporal resolution of the experiment was $152 \mathrm{fs}$. SA molecules were heated to $55^{\circ} \mathrm{C}$ in an Even-Lavie valve and expanded into a vacuum chamber with 4 bar helium as backing gas. The ejected photoelectrons were separated according to their time-of-flights by a magnetic bottle photoelectron spectrometer.

\section{Results and Discussion}

In this work, we found a drastic dependence of the photo-induced dynamics of SA on the excitation energies. Fig. 2 shows the decay of the integrated photoelectron-count with kinetic energies between 1.0 and $1.2 \mathrm{eV}$. Upon excitation by $370-\mathrm{nm}$ photons, a prominent decay faster than the time resolution was observed. This decay can be attributed to the proton transfer process. On the other

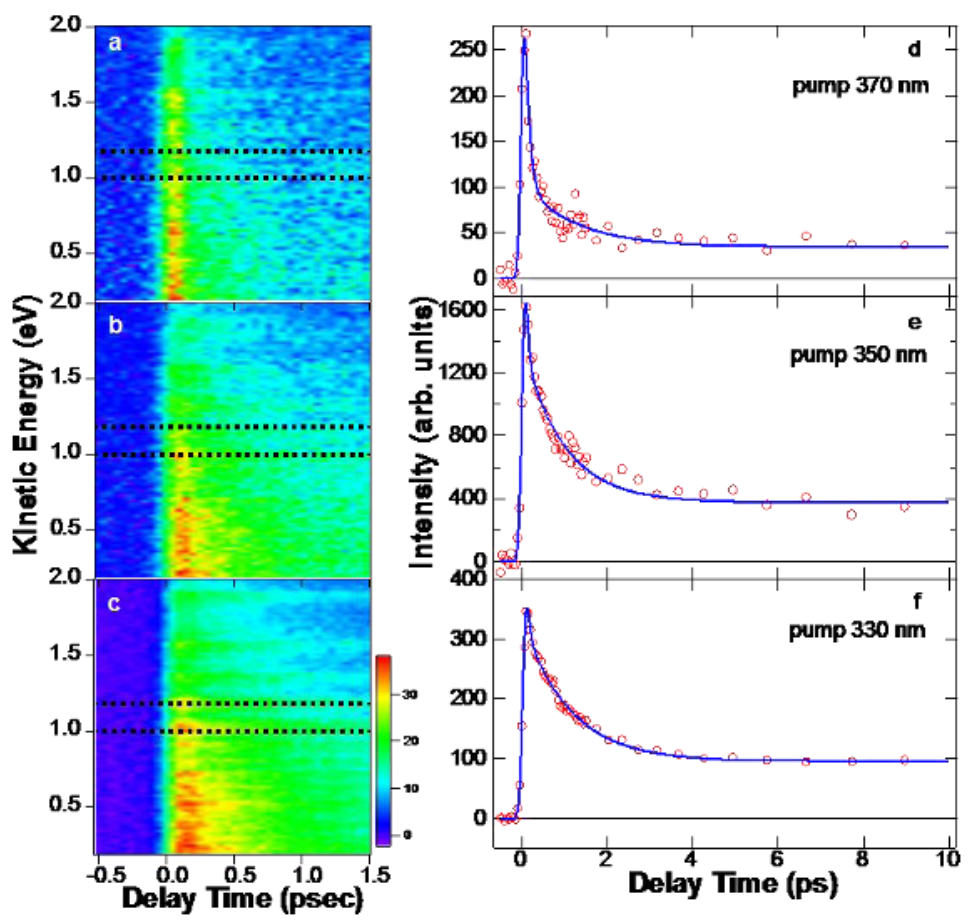

Fig. 2: Time-resolved photoelectron spectra of $N$-salicylideneaniline at pump wavelengths of a) 370 , b) 350 , and c) $330 \mathrm{~nm}$. The probe wavelength was $400 \mathrm{~nm}$ in all cases. In the right panel, the corresponding time dependences of the photoelectron yield between 1.0 and $1.2 \mathrm{eV}$, highlighted by the dotted lines in the left panel is depicted for probe wavelengths of d) 370 , e) 350 , and f) $330 \mathrm{~nm}$. 
hand, when the pump wavelength was shifted to 350 and further to $330 \mathrm{~nm}$, the initial decay time was lengthened to $1.1 \mathrm{ps}$. These features were observed in all energy regions between 0.1 and $2 \mathrm{eV}$. To elucidate the drastic change in the decay time, the energy levels of SA were calculated at B3LYP/aug-cc-pVDZ level. The following results were found: i) The aniline ring of SA is twisted in the minimum energy ground state geometry by 35 degrees. ii) In the $\mathrm{S}_{1}$ state, the planar structure is more stable. iii) Proton transfer preferably takes place in planar geometry because of an energy barrier which increases for larger twist angles of the aniline-ring.

A schematic potential energy surface as a function of twist angle of the molecule is illustrated in Fig. 3. From the calculations, the different decay dynamics can be understood as follows: $370 \mathrm{~nm}$ photons exclusively excite the planar SA, where proton transfer can happen immediately after excitation and without crossing a barrier. The lifetime of proton transfer is predicted to be less than $50 \mathrm{fs}$. On the other hand, $330 \mathrm{~nm}$ photons predominantly excite twisted SA-molecules. The decay time of $1.1 \mathrm{ps}$ roughly coincides with the period of the twisting motion needed for planarization ( 830 fs) and allowing proton transfer to take place. This example underlines the importance of planarity for proton transfer processes.

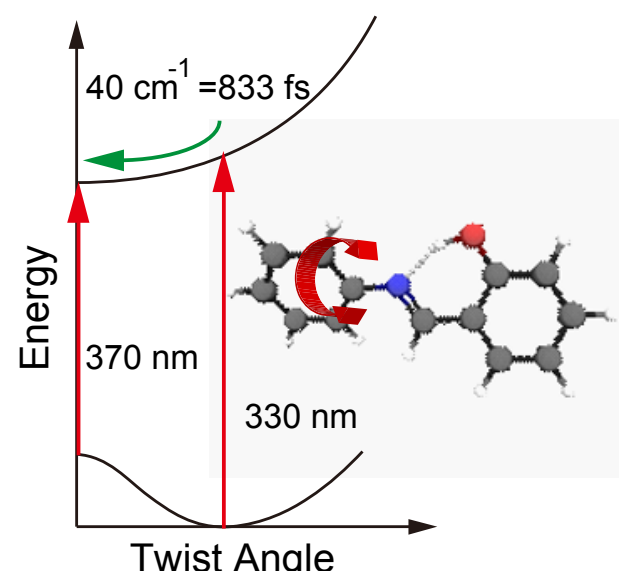

Fig. 3: Schematic illustration of the potential surface as a function of twist angle of the anil group. 370- and 330-nm photons mostly excite planar and twisted molecules, respectively. The twisting frequency in the excited state is $40 \mathrm{~cm}^{-1}$.

\section{References}

1. C. Randino, M. Ziółek, R. Gelabert, J. A. Organero, M. Gil, M. Moreno, J. M. Lluch, and A. Douhal, Phys. Chem. Chem. Phys. 13, 14960 (2011). 